pressibility and Bernoulli's theorem, to mention only a few. A brief account is included of the gas laws, with which one is obviously concerned when designing accumulators or air bottles.

Next Mr. Parker and Mr. Conway discuss hydraulic systems and circuits. It is obviously impossible to lay down a set of rules for designing a complete system in a text-book; but in Chapters 4 and 5 various aspects, including pumps, piping, jacks and circuits, are considered and sound advice is given on both desirable and undesirable features for a system.

Finally, Mr. Black considers the installation, operation and testing of a hydraulic system in an aircraft. He very wisely emphasizes the importance of good filtration and careful attention by operating personnel to clean working methods and conditions, and in the section on fault-finding he warns the reader never to assume that a component is too simple to go wrong. Mr. Black also discusses the design and use of full-scale rigs for ground-testing complete hydraulic systems, prior to flight-testing in prototype aircraft.

Vol. 2 deals in detail with components for aircraft hydraulic systems. Separate chapters are devoted to seals, pumps, jacks, servo-controls and many other devices. Space limitations preclude a detailed review of each of the subjects treated, but it may suffice to say that they are all discussed and explained thoroughly. The book abounds with sectional drawings and diagrams of components made by both British and foreign firms; the information given is remarkably complete and represents hard-won and up-to-date experience.

These volumes cover the whole field of aircraft hydraulics and yet the individual subjects are treated so thoroughly that a very good working knowledge is obtained. Particular emphasis is placed on the practical aspects throughout, but this is backed by suitable theoretical explanations. These books can be recommended highly and should prove very valuable to students or technicians starting work in this field. They should also be useful to those more experienced specialists who may wish to refresh their memories on the other man's problems.

\section{THEORY OF SOLUTIONS}

\section{The Molecular Theory of Solutions}

By Prof. I. Prigogine, with the collaboration of A. Bellemans and V. Mathot. (Series in Physics.) Pp. $x x+448$. (Amsterdam : North-Holland Publishing Company, 1957.) 48 guilders.

TN theoretical physics it is often more useful to circumvent an intractable problem than to solve it; this is nowhere more true than in the statistical theory of solutions. In principle (as can so often be claimed) the thermodynamic properties of any mixture of molecules are determined precisely by the intermolecular potentials, and one should, therefore, be able to calculate the equation of state from a knowledge of intermolecular forces alone. In practice, however, this is a fruitless undertaking except at densities so low that a virial expansion can be performed with accuracy. Our failure to construct an exact theory of the liquid state is a sufficient indication of the hopelessness of attempting an $a$ priori theory of liquid solutions. It therefore becomes pertinent to ask : What should be the aim of theories of solutions and how are we to judge their success or utility ? Only to ask this question suggests a promising line of attack. The primary aim of solution theories must be to express in terms of the relevant intermolecular potentials the change in free energy which accompanies the mixing of pure fluids with one another. In order to yield useful results the theory of solutions must, therefore, make full use of the experimental properties of the pure components under conditions which resemble as nearly as possible the condition of the solution itself. This philosophy, in recent years, has led to notable advances, and these are fully and carefully described in Prof. I. Prigogine's book.

Of particular interest in solution theory are mixtures of molecules the intermolecular potentials of which are of the Lennard-Jones type, leading to a short-range repulsion and a long-range attraction. Pure species of this sort obey the law of corresponding states, and this law can be generalized to mixtures of Lennard-Jones molecules provided their force fields are isotropic, and do not differ too greatly in strength. The theory of such mixtures, now known as 'conformal solutions', must, however, be extended in order to describe adequately the properties of mixtures in which there are specific interactions or in which the molecules are of very different size. Prof. Prigogine and his colleagues have been largely responsible for this expansion of the theory, and have also account. ed successfully for the properties of special classes of mixtures such as associated solutions, mixtures of isotopic species and certain types of polymer solution.

The mathematical methods used in the book are sufficiently elementary to be followed by the ordinary physical chemist, and the book contains many numerical examples which enable the reader to test his understanding of the theory. The physical arguments are clearly and fully expounded and there is much valuable new material which has not yet appeared in the literature. Altogether this is a thoroughly up-to-date and critical book which can be warmly recommended to those wishing to acquaint themselves with the most important recent developments in the theory of non-electrolyte solutions.

H. C. LoNGUET-HigGrins

\section{SOLUTION OF EIGENVALUE PROBLEMS}

\section{Variational Methods for Eigenvalue Problems}

An Introduction to the Mothods of Rayleigh, Ritz, Weinstein, and Aronszajn. By Prof. S. H. Gould. (Mathematical Expositions, No. 10.) Pp. xiv +179. (Toronto: University of Toronto Press ; London : Oxford University Press, 1957.) 48s. net.

TF $H$ is a linear operator, the values of $\lambda$, real or 1 complex, for which the equation $H x=\lambda x$ has solutions in $x$ satisfying given boundary conditions, are the eigenvalues of the equation.

If the space of the elements $x$ is finite-dimensional, the eigenvalues can be obtained by standard methods; but these methods may become impossibly difficult when this space is infinite-dimensional (for example, Hilbert space), and it is then that the variational method is of great value in many practical cases. This consists in finding the minima of certain expressions associated with the above equation. If now we 